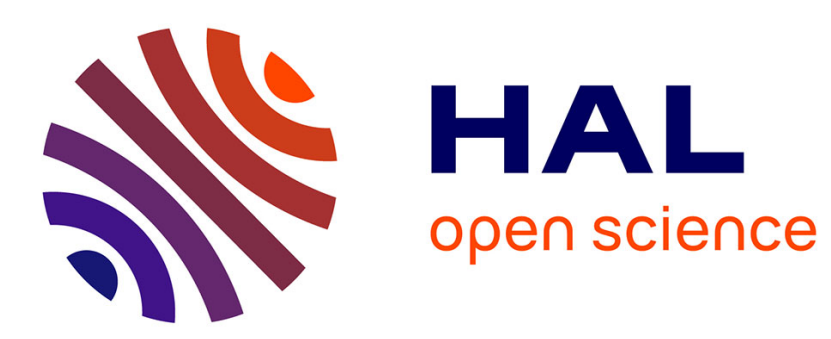

\title{
High Water Flux with Ions Sieving in a Desalination 2D Sub-Nanoporous Boron Nitride Material
}

X. Davoy, A. Gellé, Jean-Christophe Le Breton, Hervé Tabuteau, A. Soldera, Anthony Szymczyk, Aziz Ghoufi

\section{- To cite this version:}

X. Davoy, A. Gellé, Jean-Christophe Le Breton, Hervé Tabuteau, A. Soldera, et al.. High Water Flux with Ions Sieving in a Desalination 2D Sub-Nanoporous Boron Nitride Material. ACS Omega, 2018, 3 (6), pp.6305-6310. 10.1021/acsomega.8b01076 . hal-01834890

HAL Id: hal-01834890

https://hal-univ-rennes1.archives-ouvertes.fr/hal-01834890

Submitted on 17 Jul 2019

HAL is a multi-disciplinary open access archive for the deposit and dissemination of scientific research documents, whether they are published or not. The documents may come from teaching and research institutions in France or abroad, or from public or private research centers.
L'archive ouverte pluridisciplinaire HAL, est destinée au dépôt et à la diffusion de documents scientifiques de niveau recherche, publiés ou non, émanant des établissements d'enseignement et de recherche français ou étrangers, des laboratoires publics ou privés. 


\title{
High Water Flux with lons Sieving in a Desalination 2D Sub- Nanoporous Boron Nitride Material
}

\author{
Xavier Davoy, ${ }^{\dagger}$ Alain Gellé, ${ }^{\dagger}$ Jean-Christophe Lebreton, ${ }^{\dagger}$ Hervé Tabuteau, $^{\dagger}$ Armand Soldera, ${ }^{\ddagger}$ \\ Anthony Szymczyk, ${ }^{\S}$ and Aziz Ghouf*, ${ }^{\dagger}$ (])
}

†Univ Rennes,CNRS, Institut de Physique de Rennes (IPR)—UMR 6251, F-35000 Rennes, France

${ }^{*}$ Department of Chemistry, Université de Sherbrooke, Sherbrooke, Québec J1K 2R1, Canada

${ }^{\S}$ Univ Rennes, CNRS, Institut de Sciences Chimiques de Rennes (ISCR)-UMR 6226, F-35000 Rennes, France

\author{
Supporting Information
}

\begin{abstract}
Over the past decades, desalination by reverse osmosis (RO) membranes has attracted increasing attention. Although RO has proven its efficiency, it remains, however, relatively costly because of the use of highpressure pumps and the low water permeability of conventional cross-linked polymer membranes. One route to improve the desalination performance consists of using membranes made from sub-nanoporous boron nitride (sNBN) monolayers. Indeed, by using molecular dynamics simulations, we report here that the water permeability of such sNBN membranes far exceeds that of conventional RO polymer membranes and is even higher than that of nanoporous graphene while the ion rejection remains close to $100 \%$. At the same time, the molecular mechanism of water and ion transport through sNBN has been elucidated, with special attention paid to the impact of ions on water permeability through sNBN membranes.
\end{abstract}

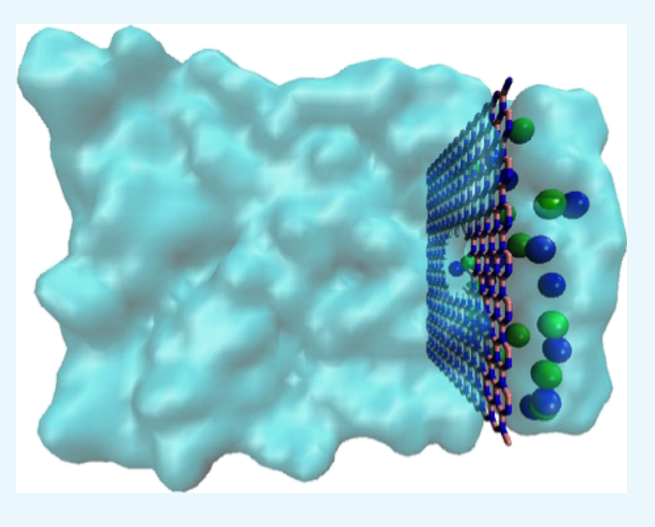

\section{INTRODUCTION}

With freshwater sources in short supply and uneven availability, efficient and sustainable technologies for water desalination have a crucial role to play in addressing the world's clean water needs in the 21 st century. ${ }^{1,2}$ Desalination is, in many regards, the most promising approach to solve the global issue of freshwater scarcity because the salted water resource on the earth is "virtually unlimited". Over the past decades, seawater desalination by reverse osmosis ( $\mathrm{RO}$ ) membranes has become a common method for countries with direct access to the sea. Although RO has proven its efficiency, it remains, however, relatively costly because of (i) seawater osmotic pressure, which requires the use of high-pressure pumps, and (ii) the low water permeability of conventional RO membranes, which requires large membrane surface areas. ${ }^{1-3}$ Recently, considerable effort has been invested in the development of new RO membranes to improve the water permeability. However, it was recently pointed out that energy savings allowed by these ultrapermeable membranes exhibit a law of diminishing returns because of thermodynamics and concentration polarization at the membrane surface leading to a limited improvement compared with the current levels of energy consumption achieved with polyamide membranes. ${ }^{2,4-6}$ For instance, it was established that a tripling in water permeability would allow for $15 \%$ less energy for a seawater RO plant with a given capacity and recovery ratio. ${ }^{5}$ Despite these limitations regarding seawater desalination, it is fundamental to capture and to understand the microscopic mechanisms ruling water and ion transport through these two-dimensional (2D) membranes and to continue our attempts to predict and to develop materials and processes that will allow optimizing membrane separations. Among popular nanoporous materials, carbon nanotube (CNT) arrays $^{7}$ have been widely investigated as potential candidates for water desalination. Both experiments and molecular dynamics (MD) simulations have demonstrated that CNTs can allow fast water flow ${ }^{8-13}$ and a salt rejection about 95\%. ${ }^{14}$ Another route to improve both salt rejection and water permeability consists of using $2 \mathrm{D}$ nanoporous membranes such as graphene sheets containing artificial (sub) nanometric holes. ${ }^{15,16}$ Water can flow across such 2D membranes thanks to its small molecular size, whereas ion passage can be blocked given the larger size of the ion hydration shell. Recently, several groups have theoretically investigated the molecular mechanisms ruling water transport and ionic selectivity through stacked sheets of graphene oxide, $^{17,18}$ nanoporous graphene, ${ }^{5,15,19-21}$ graphyne, ${ }^{2,23}$ $\mathrm{MoS}_{2}{ }^{24} \mathrm{C}_{2} \mathrm{~N}{ }^{25}$ and theoretical supersquare ${ }^{26}$ membranes. Garnier et al. have shown from MD simulations that the water flux through sub-nanoporous boron nitride ( $\mathrm{sNBN}$ ) monolayer membranes is expected to be higher than that through nanoporous graphene monolayers, thus heralding that $2 \mathrm{D}$ boron nitride $(\mathrm{BN})$ could be an attractive candidate for the next

Received: $\quad$ May 21, 2018

Accepted: May 28, 2018

Published: June 12, 2018 
generation of nanofilters. ${ }^{27}$ More recently, Srivastava et al. showed the potential applicability of $\mathrm{BN}$ as an efficient adsorbent for the removal of arsenic ions. ${ }^{28}$ In comparison with carbon-based materials, $\mathrm{BN}$ exhibits higher chemical stability, improved biocompatibility, higher Young modulus, and better resistance to oxidation at high temperatures. Furthermore, BN nanotubes have shown superior water permeability performance compared with CNTs of similar diameter and length, ${ }^{29,30}$ which has been attributed to frictionless transport. ${ }^{12,13,25,31}$ To date, however, desalination performance of $2 \mathrm{D}$ sNBN membranes has never been investigated. In this work, we performed $\mathrm{MD}$ simulations in order to predict sodium chloride rejection and water permeability of four $\mathrm{sNBN}$ membranes having different pore sizes and geometries, namely, two hydrogenated circular pores with two different sizes (hereinafter referred to as sNBN@1 and sNBN@3), a dehydrogenated circular pore (hereinafter referred to as sNBN@4), and a dehydrogenated triangular pore (hereinafter referred to as sNBN@2).sNBN@2 and sNBN@4 are not hydrogenated given the constrained geometry and the small pore radius that prevent the hydrogen grafting. The choice of investigating a triangular pore was motivated by the existence of natural triangle-shaped defects in hexagonal $\mathrm{BN}$ monolayers. $^{32,33}$ Given the difficulty to generate nanopores with a controlled size, ${ }^{16}$ it would then be relevant to use these defects as selective pores. ${ }^{32,33}$ An illustration of the four different membranes is provided in Figure 1. Pores with surface

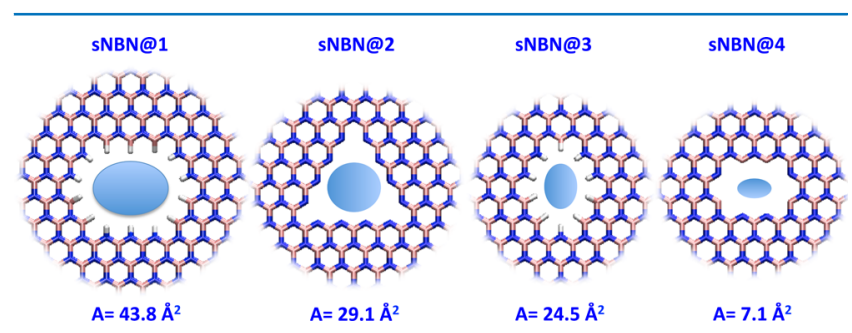

Figure 1. Illustration of the four sNBN membranes (the pore surface area (A) is indicated for each membrane).sNBN@1 and sNBN@3 are hydrogenated pores, whereas sNBN@2 and sNBN@4 are dehydrogenated pores. Boron, nitrogen, and hydrogen atoms are shown in pink, blue and, white colors, respectively. areas of the same order of magnitude compared to those of the (sub-nanometric) natural defects in BN were considered. The pore surface area was calculated by rolling a nitrogen molecule probe along the pore surface. The surface area of a nanopore was calculated by a difference between the accessible surface area of nonporous monolayer and the accessible surface area of porous membrane. Calculation of accessible surface area is detailed in the Supporting Information. Force field and computational procedure are described in the Methods section. The relevance of the water-BN force field parameters was checked by comparing the contact angle of a water droplet on a $\mathrm{BN}$ monolayer computed from $\mathrm{MD}$ with experimental data. Details of simulations are given in the Methods section. A predicted contact angle of $55^{\circ}$ was thus found, which is in good agreement with the experiment $\left(40-60^{\circ}\right) \cdot{ }^{34}$ As illustrated in Figure 2a, pressure-driven transport properties of $\mathrm{sNBN}$ monolayers were investigated by carrying out nonequilibrium MD (NEMD) simulations, where two rigid pistons were used to generate a pressure difference $(\Delta P)$ across the sNBN membrane. The protocol was specified in the Methods section.

\section{RESULTS AND DISCUSSION}

Water Permeability. In Figure $2 b$, we report the water permeability $\left(P_{\mathrm{w}}\right)$ of the four membranes as a function of $\Delta P$ for pure water and $1 \mathrm{M} \mathrm{NaCl}$ solutions. $P_{\mathrm{w}}$ was calculated by counting the number of water molecules $\left(N_{\mathrm{w}}\right)$ crossing the membrane as a function of time. Data were analyzed at steady state, that is, when $N_{\mathrm{w}}$ was found to vary linearly with time (see Figure S1 of the Supporting Information). Water transport across $2 \mathrm{D}$ porous materials is influenced by both the interfacial interactions and the friction between the water molecules and the membrane material. ${ }^{12,13}$ Tocci et al. highlighted the very weak friction between water molecules and hexagonal $\mathrm{BN}^{35}$ which favors sliding and transport of water molecules across sNBN pores. As expected, sNBN@1 and sNBN@2, with the two highest pore surface areas (see Figure 1), exhibit the highest pure water permeabilities. Strikingly, the narrowest pore, sNBN@4, is found to be more permeable to pure water than the widersNBN@3 pore. This finding indicates that the discrepancy between the water permeability of the various sNBN membranes cannot be explained solely by the different pore sizes. As depicted in Figure 3a, which shows the 2D

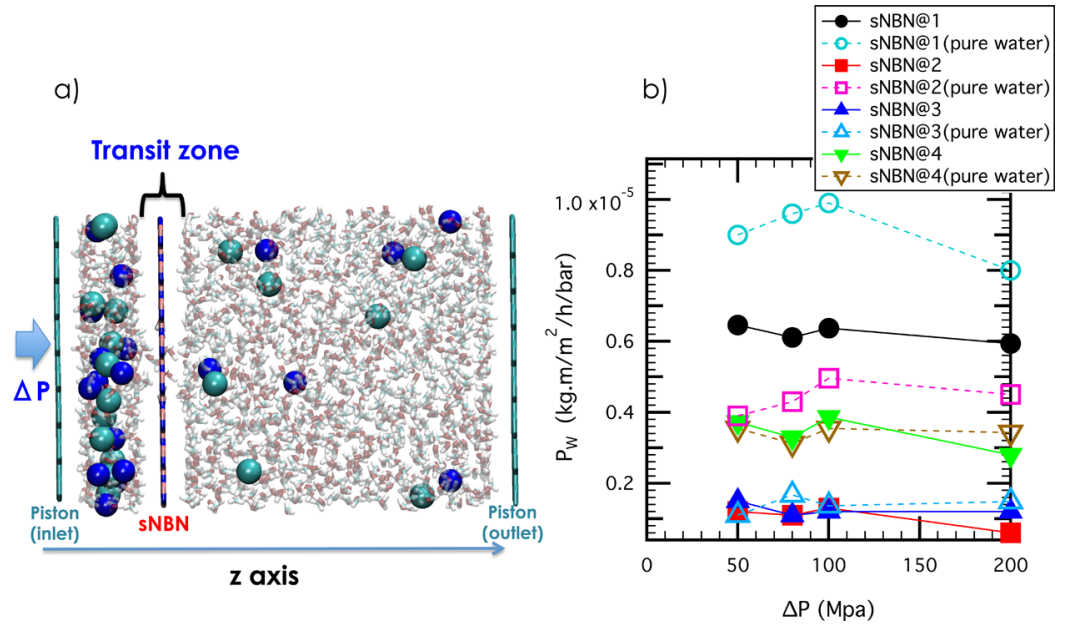

Figure 2. (a) Illustration of a final configuration. Cyan and blue colors correspond to the $\mathrm{Cl}^{-}$and $\mathrm{Na}^{+}$ions. (b) Water permeability of the four sNBN membranes as a function of the transmembrane pressure difference $(\Delta P)$. 


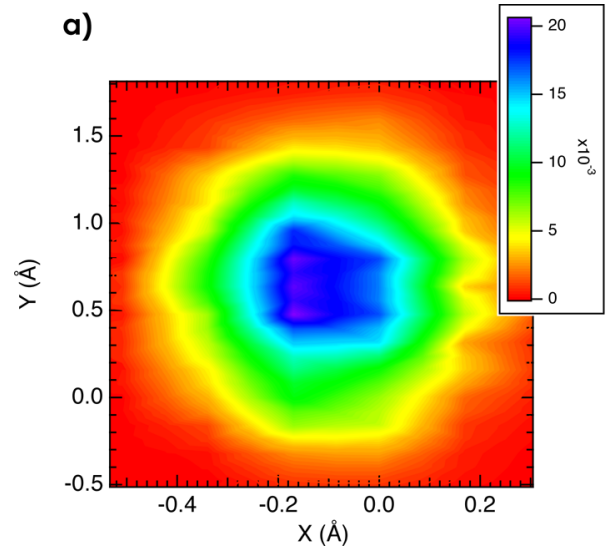

b)

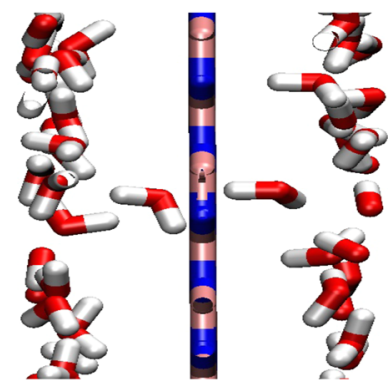

Figure 3. (a) Two-dimensional density profiles (along the $x$ and $y$ directions) of water molecules located in the positive transit zone (i.e., between $z$ $=0$ and $3 \AA$ ) for the sNBN@4 membrane. (b) Illustration of single-file transport of water molecules through the sNBN@4 membrane.
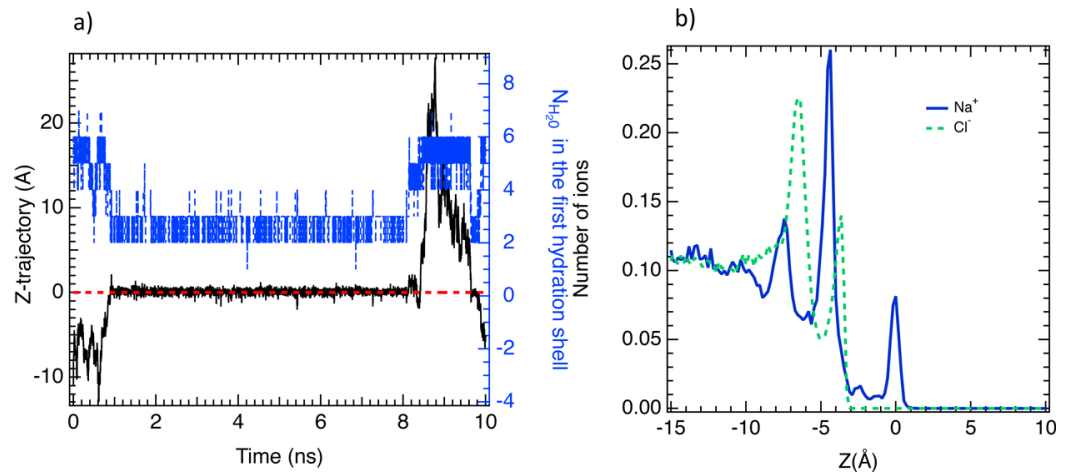

Figure 4. (a) Trajectory of a $\mathrm{Na}^{+}$ion along the $z$ axis (black line and left axis) and its hydration number (blue line and right axis) at $\Delta P=100 \mathrm{MPa}$ through the sNBN@2 membrane, which is located at $z=0 \AA$ (dashed red line). (b) Density profile of ions along the $z$ axis for sNBN@2.

density profile of water close to sNBN@4, the size of the water density pattern indicates single-file transport across this membrane (see the illustration in Figure $3 \mathrm{~b}$ ). It results from the ultraconstrained geometry where water molecules can be transferred only one at a time. Such a transport mode was not observed for the other sNBN membranes with wider pores. The single-file transport leads to a less cohesive hydrogen-bond (HB) network of water molecules across sNBN@4 than across the other membranes. For instance, the calculation of the number of $\mathrm{HBs}(n \mathrm{HB})^{36}$ between water molecules in the transit zone (which is defined in Figure 2a between $z=-3 \AA$ and $z=3$ $\AA$ ) leads to $n \mathrm{HB}=1.1 \pm 0.2$ and $n \mathrm{HB}=2.2 \pm 0.2$ for sNBN@4 and sNBN@1, respectively, which corroborates single-file transport mechanism through sNBN@4. As shown in Figure $2 \mathrm{~b}$, the addition of salt leads to a decrease in $P_{\mathrm{w}}$ for sNBN@1 andsNBN@2. It results from the additional resistance to water transfer caused by ions during their translocation through the pore. It is illustrated in Figure 4, which shows a sodium cation "blocked" inside the sNBN@2 pore, thus leading to a substantial hindrance to water permeability (see also the movie provided in the Supporting Information). The impact of salt on the water permeability is found to be weaker for sNBN@1 because of its wider surface area $\left(43.8 \AA^{2}\right)$, which allows ions to be transferred with their full first hydration shell (see Figure S2 of the Supporting Information) without significant hindrance to water flow. Indeed, the average distance between $\mathrm{Na}^{+}$and the oxygen atoms of water molecules in the sNBN@1 pore is about $3.3 \AA$, which highlights that these water molecules belong to the first hydration shell of $\mathrm{Na}^{+}$(a similar qualitative behavior was observed for $\mathrm{Cl}^{-}$ions). Interestingly, we also report in Figure S3 of the Supporting Information the trajectory of a water molecule and its hydration number in the first shell through sNBN@1. Figure S3 shows that the water molecule also conserves its hydration shell during the translocation given the size of pore. Strikingly, water permeability is found to be almost unaffected by the presence of ions for sNBN@4 (see Figure 2b). Actually, the very small pore of sNBN@4 and sNBN@3 prevents the passage of hydrated ions, and the high energetic penalty associated with ion dehydration results in a total ion rejection by sNBN@4 (see below). Ions cannot enter the pore and then have a much less impact on water permeability compared to the other membranes with wider pores (note that single-file transport was still observed for sNBN@4 in the presence of ions). It is noteworthy that the impact of ion translocation on water permeability through sNBN membranes is so strong that, in the presence of ions, the water permeability of sNBN@4 is greater than that of sNBN@2, whereas the opposite is true when dealing with pure water (see Figure 2b). As for sNBN@1, we report in Figure S4 of the Supporting Information the trajectory of a water molecule and its hydration number in the first shell throughsNBN@4. Contrary to sNBN@1, the water molecule undergoes a loss of two water molecules during the passage through the sub-nanopore. This provides a microscopic picture allowing a better understanding of the improved performance of the sNBN@4 membrane.

Ion Rejection. We report in Figure 5 the rejection rate of 1 $\mathrm{M} \mathrm{NaCl}$ solutions as a function of water permeability for 


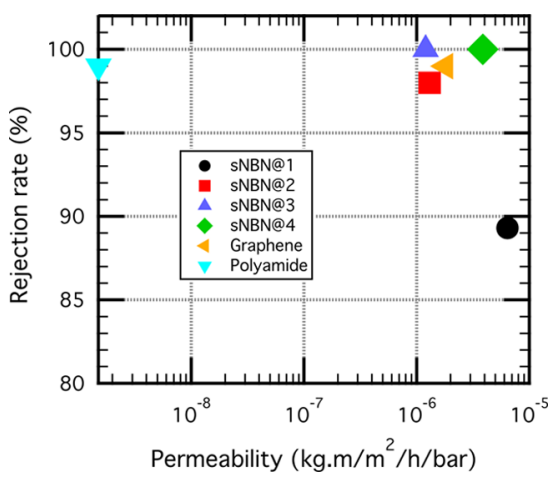

Figure 5. Rejection rate of a $1 \mathrm{M} \mathrm{NaCl}$ solution by different membranes vs water permeability. Data reported for sNBN membranes were obtained for $\Delta P=100 \mathrm{MPa}$. Surface area of hydrogenated graphene is about $65.6 \AA^{2}$. Results on porous graphene and polyamide membranes were obtained from our own $\mathrm{MD}$ simulations. Force field of polyamide and nanoporous graphene can be found elsewhere. ${ }^{27,37}$

various kinds of membranes. The rejection rates were calculated from the number of ions and water molecules crossing the membrane, $\left(1-c_{\text {outlet }} / c_{\text {inlet }}\right) \times 100$, where $c_{\text {outlet }}$ and $c_{\text {inlet }}$ are the concentrations in the low- and high-pressure reservoirs, respectively (see Figure 2a). It is worth mentioning that all simulation results reported in Figure 5 were obtained with the same water model, TIP $4 \mathrm{P} / 2005$, and that additional simulations performed with another water model (SPC) led to qualitatively similar results, thus indicating that the conclusions drawn hereafter are not force field-dependent. For $\Delta P=100 \mathrm{MPa}$, water permeability for the SPC water model through sNBN@4 was found to be equal to $4.1 \times 10^{-6}$ $\mathrm{kg} \cdot \mathrm{m} / \mathrm{m}^{2} / \mathrm{h} / \mathrm{bar}$, which is close to the value obtained from TIP4P/2005 $\left(3.9 \times 10^{-6} \mathrm{~kg} \cdot \mathrm{m} / \mathrm{m}^{2} / \mathrm{h} / \mathrm{bar}\right)$. The rejection rates by sNBN@2,sNBN@3, and sNBN@4 were found to be similar and close to $100 \%$ whatever $\Delta P$. It can be explained by the small pore size of these membranes, which prevents hydrated ion translocation. Because sNBN@4 has the highest water permeability, it therefore exhibits the best desalination performance among the various sNBN membranes under consideration. As shown in Figure 5, water permeability through sNBN membranes was found to exceed that of usual RO polyamide membranes ${ }^{37}$ by several orders of magnitude. The water permeabilities of sNBN@1 and sNBN@4 were even higher than that of nanoporous graphene (another new promising $2 \mathrm{D}$ nanofilter). It should be stressed, however, that ions were only partially rejected by sNBN@1 because the effective surface area of hydrated ions (about $38.5 \AA^{2}$ for $\mathrm{Na}^{+}$) was substantially lower than the sNBN@1 pore surface area (see Figure 1), thus allowing ions to be transferred across the membrane, contrary to the sNBN@2,sNBN@3, and sNBN@4 membranes for which pore surface areas were smaller than those of hydrated ions. As shown in Figure 4a, sodium cations undergo partial dehydration when crossing the sNBN@2 membrane. Indeed, the hydration number of $\mathrm{Na}^{+}$in the vicinity of the pore entrance is found to be between 2 and 3, which indicates a substantial dehydration because the hydration number was found to be 5-6 far from the membrane. The dehydration process is of course unfavorable from the thermodynamic point of view, which explains high salt rejection (about 98\% for sNBN@2 and almost 100\% for both sNBN@3 and sNBN@4). Interestingly, Figure 4a shows that with sNBN@2, ions dwell in to the nanopore for $6 \mathrm{~ns}$ before being translocated. As shown in Figure $4 b$, where the profile of densities of ions is plotted, a maximum in the density of $\mathrm{Na}^{+}$is shown exactly at the position of sNBN@4 $(z=0 \AA)$, which shed light on the fact the ions dwell the nanopore.

\section{CONCLUSIONS}

To sum up, the present work highlights the outstanding separation performance of $\mathrm{sNBN}$ monolayers. The best performance was obtained with the sNBN@4 membrane. It results from the combination of a small pore size with specific interfacial properties, which prevents translocation of (hydrated) ions while allowing fast water transport due to sliding of a water wire (single-file transport). Molecular simulations performed with pure water and sodium chloride solutions allowed us to provide a microscopic picture of the impact of ions on water permeability through sNBN membranes. Finally, the energetically unfavorable ion dehydration was shown to result in high salt rejection. Although the present results were obtained from molecular simulations, recent experimental works suggest that large-scale sNBN membranes could be produced with reasonable efforts. For instance, sNBN membranes could be synthesized from oxygen-plasma etching ${ }^{16}$ or by electrically accentuating the natural defects in $\mathrm{BN}$ monolayers (i.e., by taking advantage of the presence of defects). 32,33

\section{METHODS}

sNBN membranes were made by removing few atoms at the center of BN monolayer. For sNBN@1 and sNBN@3, hydrogen atoms have been added to keep the usual valence of carbon. The partial charges on boron and nitrogen atoms were calculated from the first-principles calculations. Details of calculations can be found elsewhere ${ }^{38}$ Partial charges on each pore are given in file FIELD.txt whereby box dimensions, coordinates, partial charges, and Lennard-Jones parameters of atoms of four membranes are provided. The sNBN membranes were considered as rigid. Water was modeled by using the nonpolarizable rigid TIP4P/2005 model, ${ }^{39}$ and a nonpolarizable force field was considered for $\mathrm{Na}^{+}$and $\mathrm{C}^{-} \cdot{ }^{40}$ Indeed, it has been shown that the polarizability of $\mathrm{Na}^{+}$and $\mathrm{Cl}^{-}$had a negligible impact on both their structure and dynamics properties. $^{41}$ NEMD simulations were conducted by using two pistons (graphene walls), on which a given pressure was applied. The force to be applied on the piston atoms was calculated from the relation $F=\Delta P S / N_{\mathrm{p}}$, where $\Delta P$ is the pressure difference, $S$ is the piston surface area, and $N_{\mathrm{p}}$ is the piston atom number. The sNBN membrane was located at the center of the simulation box $(z=0 \AA)$ and was surrounded by two water reservoirs containing 1800 water molecules. A $1 \mathrm{M}$ $\mathrm{NaCl}$ solution $\left(20 \mathrm{Na}^{+}\right.$and $20 \mathrm{Cl}^{-}$ions) was initially located in only one of the two reservoirs. Let us mention that the solid/ liquid interface was along the $z$ direction (see Figure 2a). The flow was therefore along the $z$ direction. NEMD simulations were stopped when the water density in the inlet reservoir was constant. To be reproducible, five simulations with different initial configurations have been considered. During the pore filling, the number of molecules increases and stabilizes to reach a plateau with a fluctuation of 5-8 water molecules. All simulations were carried out with the DLPOLY package (version 4.0 $)^{42}$ using the velocity-Verlet algorithm in the NVT statistical ensemble, where $N$ is the particle number, $V$ is the volume, and $T$ is the temperature. The Berendsen thermostat ${ }^{43}$ 
was considered. Periodic boundary conditions were applied in the three directions. Simulations were performed at $T=300 \mathrm{~K}$ using a time step of 0.001 ps to sample $10 \mathrm{~ns}$ (acquisition phase). Electrostatic interactions were truncated at $12 \AA$ and calculated by using the Ewald sum method with a precision of $10^{-6}$. Short-range interactions were modeled by using a Lennard-Jones potential with a cutoff of 12 Å. Lennard-Jones interactions between the solid frameworks and water molecules were taken into account by means of the Lorentz-Berthelot mixing rule. The coordinates for the various membranes (CONFIG files), the force field (FIELD files), and the input parameters (CONTROL file) of simulations are provided. These files are the input files of DL_POLY software.

The calculation of the contact angle was performed by depositing a water droplet on a $\mathrm{BN}$ membrane made of four monolayers to compare with data reported in the literature. ${ }^{34} \mathrm{~A}$ water droplet of 2184 molecules (a radius of $22 \AA$ ) was carved from a liquid phase (a system made of 4000 water molecules and simulated in the isothermal-isobaric statistical ensemble at 1 bar and $298.15 \mathrm{~K}$ for $10 \mathrm{~ns}$ ). After deposit, MD simulations in the canonical ensemble were performed for 1 ns. $\Phi$ was evaluated from the 2D density profile according to the $z$ and $r=$ $(x+y)^{0.5}$ directions. ${ }^{34} \mathrm{~A}$ value of $55^{\circ}$ was found, which is in good agreement with recent works whereby parameters interactions were optimized ${ }^{34}$ and then validates the crossed interactions between $\mathrm{BN}$ and water considered in this work.

\section{ASSOCIATED CONTENT}

\section{S Supporting Information}

The Supporting Information is available free of charge on the ACS Publications website at DOI: 10.1021/acsomega.8b01076.

Coordinates for the various membranes, the force field, and the input parameters of simulations (ZIP)

Filtered water molecule number through the sNBN membranes, illustration of $\mathrm{Na}^{+}$and water molecules in the pore of the sNBN@1 membrane, trajectory of a water molecule along the $z$ axis in sNBN@1, and trajectory of a water molecule along the $z$ axis in sNBN@ 4 (PDF)

Movie showing the simulations to predict sodium chloride rejection and water permeability (MPG)

\section{AUTHOR INFORMATION}

\section{Corresponding Author}

*E-mail: aziz.ghoufi@univ-rennes1.fr.

ORCID 1

Armand Soldera: 0000-0001-5467-5714

Anthony Szymczyk: 0000-0001-7298-3606

Aziz Ghoufi: 0000-0003-0877-7968

Notes

The authors declare no competing financial interest.

\section{ACKNOWLEDGMENTS}

The authors are grateful to (i) the University of Rennes 1 for its financial support through the program "Défis émergents 2017", (ii) the CNRS for its financial support through the program "PEPS Infinity" and the PICS "Waterloo", and (iii) Total SA and the CNRS for their financial support through the program "PEPS Boomer".

\section{REFERENCES}

(1) Miller, J. Review of Water Resources and Desalination Technologies, 2003.

(2) Elimelech, M.; Phillip, W. A. The Future of Seawater Desalination: Energy, Technology, and the Environment. Science 2011, 333, 712 .

(3) Shannon, M. A.; Bohn, P. W.; Elimelech, M.; Georgiadis, J. G.; Mariñas, B. J.; Mayes, A. M. Science and technology for water purification in the coming decades. Nature 2008, 452, 301.

(4) Song, L.; Hu, J. Y.; Ong, S. L.; Ng, W. J.; Elimelech, M.; Wilf, M. Performance limitation of the full-scale reverse osmosis process. J. Membr. Sci. 2002, 214, 239.

(5) Cohen-Tanugi, D.; Grossman, J. C. Mechanical Strength of Nanoporous Graphene as a Desalination Membrane. Nano Lett. 2014, 11,6171 .

(6) Werber, J. R.; Osuji, C. O.; Elimelech, M. Materials for nextgeneration desalination and water purification membranes. Nat. Rev. Mater. 2016, 1, 16018.

(7) Fornasiero, F.; Park, H. G.; Holt, J. K.; Stadermann, M.; Grigoropoulos, C. P.; Noy, A.; Bakajin, O. Ion exclusion by sub-2-nm carbon nanotube pores. Proc. Natl. Acad. Sci. U.S.A. 2008, 105, 17250.

(8) Hummer, G.; Rasaiah, J. C.; Noworyta, J. P. Water conduction through the hydrophobic channel of a carbon nanotube. Nature 2001, 414, 188.

(9) Kalra, A.; Garde, S.; Hummer, G. Osmotic water transport through carbon nanotube membranes. Proc. Natl. Acad. Sci. U.S.A. 2003, 100, 10175.

(10) Holt, J. K. Fast Mass Transport Through Sub-2-Nanometer Carbon Nanotubes. Science 2006, 312, 1034.

(11) Falk, K.; Sedlmeier, F.; Joly, L.; Netz, R. R.; Bocquet, L. Molecular Origin of Fast Water Transport in Carbon Nanotube Membranes: Superlubricity versus Curvature Dependent Friction. Nano Lett. 2010, 10, 4067.

(12) Secchi, E.; Marbach, S.; Niguès, A.; Stein, D.; Siria, A.; Bocquet, $\mathrm{L}$. Massive radius-dependent flow slippage in carbon nanotubes. Nature 2016, 537, 210.

(13) Michaelides, A. Nanoscience: Slippery when narrow. Nature 2016, 537, 171.

(14) Thomas, M.; Corry, B. A computational assessment of the permeability and salt rejection of carbon nanotube membranes and their application to water desalination. Philos. Trans. R. Soc., A 2016, 374, 20150020.

(15) Cohen-Tanugi, D.; Grossman, J. C. Water Desalination across Nanoporous Graphene. Nano Lett. 2012, 12, 3602.

(16) Surwade, S. P.; Smirnov, S. N.; Vlassiouk, I. V.; Unocic, R. R.; Veith, G. M.; Dai, S.; Mahurin, S. M. Water desalination using nanoporous single-layer graphene. Nat. Nanotechnol. 2015, 10, 459.

(17) Chen, L.; Shi, G.; Shen, J.; Peng, B.; Zhang, B.; Wang, Y.; Bian, F.; Wang, J.; Li, D.; Qian, Z.; et al. Nature 2017, 550, 380.

(18) Abraham, J.; Vasu, K. S.; Williams, C. D.; Gopinadhan, K.; Su, Y.; Cherian, C. T.; Dix, J.; Prestat, E.; Haigh, S. J.; et al. Nat. Nanotechnol. 2017, 12, 546.

(19) Nair, R. R.; Wu, H. A.; Jayaram, P. N.; Grigorieva, I. V.; Geim, A. K. Unimpeded Permeation of Water Through Helium-Leak-Tight Graphene-Based Membranes. Science 2012, 335, 442.

(20) Wang, E. N.; Karnik, R. Water desalination: graphene cleans up water. Nat. Nanotechnol. 2012, 7, 552.

(21) Koh, D.-Y.; Lively, R. P. Nanoporous graphene: Membranes at the limit. Nat. Nanotechnol. 2015, 10, 385.

(22) Zhu, C.; Li, H.; Zeng, X. C.; Wang, E. G.; Meng, S. Quantized Water Transport: Ideal Desalination through Graphyne-4 Membrane. Sci. Rep. 2013, 3, 3163.

(23) Kou, J.; Zhou, X.; Lu, H.; Wu, F.; Fan, J. Graphyne as the membrane for water desalination. Nanoscale 2014, 6, 1865.

(24) Heiranian, M.; Farimani, A. B.; Aluru, N. R. Water desalination with a single-layer MoS2 nanopore. Nat. Commun. 2015, 6, 8616.

(25) Yang, Y.; Li, W.; Zhou, H.; Zhang, X.; Zhao, M. Tunable C2N Membrane for High Efficient Water Desalination. Sci. Rep. 2016, 6, 19218. 
(26) Sun, L.; He, X.; Lu, J. Super square carbon nanotube network: A new promising water desalination membrane. npj Comput. Mater. 2016, 2, 16004 .

(27) Garnier, L.; Szymczyk, A.; Malfreyt, P.; Ghoufi, A. Physics behind Water Transport through Nanoporous Boron Nitride and Graphene. J. Phys. Chem. Lett. 2016, 7, 3371.

(28) Srivastava, R.; Kommu, A.; Sinha, N.; Singh, J. K. Removal of arsenic ions using hexagonal boron nitride and graphene nanosheets: a molecular dynamics study. Mol. Simul. 2017, 43, 985.

(29) Won, C. Y.; Aluru, N. R. Water Permeation through a Subnanometer Boron Nitride Nanotube. J. Am. Chem. Soc. 2007, 129, 2748.

(30) Hilder, T. A.; Gordon, D.; Chung, S.-H. Boron nitride nanotubes selectively permeable to cations or anions. Small 2009, 5, 2870.

(31) Ghoufi, A.; Szymczyk, A.; Malfreyt, P. Ultrafast diffusion of Ionic Liquids Confined in Carbon Nanotubes. Sci. Rep. 2016, 6, 28518.

(32) Jin, C.; Lin, F.; Suenaga, K.; Iijima, S. Fabrication of a Freestanding Boron Nitride Single Layer and Its Defect Assignments. Phys. Rev. Lett. 2009, 102, 195505.

(33) Meyer, J. C.; Chuvilin, A.; Algara-Siller, G.; Biskupek, J.; Kaiser, U. Selective sputtering and atomic resolution imaging of atomically thin boron nitride membranes. Nano Lett. 2009, 9, 2683.

(34) Wu, Y.; Wagner, L. K.; Aluru, N. R. Hexagonal boron nitride and water interaction parameters. J. Chem. Phys. 2016, 144, 164118.

(35) Tocci, G.; Joly, L.; Michaelides, A. Friction of Water on Graphene and Hexagonal Boron Nitride from Ab Initio Methods: Very Different Slippage Despite Very Similar Interface Structures. Nano Lett. 2014, 14, 6872.

(36) Luzar, A.; Chandler, D. Effect of Environment on Hydrogen Bond Dynamics in Liquid Water. Phys. Rev. Lett. 1996, 76, 928.

(37) Ding, M.; Szymczyk, A.; Ghoufi, A. On the structure and rejection of ions by a polyamide membrane in pressure-driven molecular dynamics simulations. Desalination 2015, 368, 76.

(38) Ghoufi, A.; Deschamps, J.; Maurin, G. Theoretical Hydrogen Cryostorage in Doped MIL-101(Cr) Metal-Organic Frameworks. J. Phys. Chem. C 2012, 116, 10504.

(39) Abascal, J. L. F.; Vega, C. A general purpose model for the condensed phases of water: TIP4P/2005. J. Chem. Phys. 2005, 123, 23505.

(40) Mountain, R. G. Solvation structure of ions in water. Int. J. Thermophys. 2007, 28, 536.

(41) Zhu, H.; Ghoufi, A.; Szymczyk, A.; Balannec, B.; Morineau, D. Anomalous Dielectric Behavior of Nanoconfined Electrolytic Solutions. Phys. Rev. Lett. 2013, 109, 107801.

(42) Todorov, I.; Smith, W.; Trachenko, K.; Dove, M. T. DLPOLY3: new dimensions in molecular dynamis simulations via massive parallelism. J. Mater. Chem. 2006, 16, 1911.

(43) Berendsen, H. J. C.; Postma, J. P. M.; van Gunsteren, W. F.; DiNola, A.; Haak, J. R. Chem. Phys. 1984, 81, 3684. 